\title{
Analysis Of Re-Hospitalizations For Patients With Heart Failure Caused By Coronary Heart Disease: Data Of First Event And Recurrent Event
}

This article was published in the following Dove Press journal:

Therapeutics and Clinical Risk Management

\author{
Jing Tian (D) \\ Jingjing Yan $^{2}$ \\ Qing Zhang ${ }^{\prime}$ \\ Hong Yang ${ }^{2}$ \\ Xinlong Chen' \\ Qiang $\operatorname{Han}^{2}$ \\ Rui Han' \\ Jia Ren' \\ Yanbo Zhang ${ }^{2,3}$ \\ Qinghua Han' \\ 'Department of Cardiology, The Ist \\ Hospital of Shanxi Medical University, \\ Taiyuan, Shanxi Province 03000I, People's \\ Republic of China; ${ }^{2}$ Department of \\ Health Statistics, School of Public Health, \\ Shanxi Medical University, Taiyuan, Shanxi \\ Province 03000I, People's Republic of \\ China; ${ }^{3}$ Shanxi Provincial Key Laboratory \\ of Major Diseases Risk Assessment, \\ Taiyuan, Shanxi Province 03000I, People's \\ Republic of China
}

Correspondence: Qinghua Han Department of Cardiology, The Ist Hospital of Shanxi Medical University, 85 South Jiefang Road, Taiyuan, Shanxi Province 03000I,

People's Republic of China

$\mathrm{Tel}+86310011303$ I

Fax +86 35I 4867I46

Email syhqn@sohu.com

Yanbo Zhang

Department of Health Statistics, School of Public Health, Shanxi Provincial Key Laboratory of Major Diseases Risk

Assessment, Shanxi Medical University, 56

South Xinjian Road, Taiyuan, Shanxi Province 03000I, People's Republic of China

$\mathrm{Tel} / \mathrm{fax}+86354398505 \mathrm{I}$

Email sxmuzyb@I26.com
Background: The re-hospitalization rate of patients with heart failure remains at a high level, and studies of the subject have focused mainly on event-time outcomes. In addition to using re-hospitalization data with the outcomes of the event-time-count, this study introduces the conditional frailty model, which could help obtain more reasonable results.

Materials and methods: This prospective observational cohort study enrolled 1484 patients with heart failure caused by coronary heart disease. The outcomes of heart failure readmissions and the case report form data were collected. Based on the traditional Cox model with event-time outcomes, the mixed effects of a conditional frailty model were added to analyze the event-time-count longitudinal data.

Results: The Cox regression model showed that non-manual work, diastolic dysfunction, and better medical compensation increased the risk of heart failure readmission, whereas treatment with beta-blockers decreased the risk. The conditional frailty model further revealed that age, female sex, non-manual work, better medical compensation, longer QRS duration, and treatment with percutaneous coronary intervention increased the risk of heart failure readmission.

Conclusion: This study obtained more reliable, reasonable results based on longitudinal data and a mixed model. The results could provide more clinical epidemiological evidence for the management of heart failure.

Keywords: heart failure, coronary heart disease, re-hospitalization, conditional frailty model

\section{Introduction}

As a leading cause of heart failure (HF) worldwide, coronary heart disease (CHD) continues to be associated with a high level of morbidity and mortality. ${ }^{1}$ Patients with HF caused by CHD suffer from poor physical function and prolonged disease duration, which leads to multiple hospitalizations. Re-hospitalization is a major issue for patients with $\mathrm{HF}$ as it could increase the risk of death, result in a poor quality of life, and become a huge economic burden. ${ }^{2-4}$ Seeking effective measures to reduce readmission has become an important objective of HF management. Therefore, it is of great significance to obtain an accurate assessment of re-hospitalization.

In previous studies, re-hospitalization was mostly analyzed as time-event data, ignoring its repeatability and the counts it occurs. Moreover, there is a lack of effective, appropriate analytical methods, so prior evaluations of re-hospitalization were inappropriate and unable to predict with any accuracy the readmission of patients with HF. ${ }^{5,6}$ In this study, we introduce the "conditional frailty model" 
which includes the event-time-count data of longitudinal observations based on the Cox regression model. With the assistance of this more suitable statistical method, ${ }^{7,8}$ we could obtain more reasonable results for HF re-hospitalization, so as to provide more epidemiological evidence for HF management.

\section{Methods}

This was a multi-center, prospective cohort study to evaluate HF re-hospitalization in patients with HF caused by CHD. From 2014 to 2017, 1484 patients were enrolled according to inclusion and exclusion criteria from two medical centers in Shanxi province of People's Republic of China. The study was conducted according to the Declaration of Helsinki and approved by IRB of Shanxi Medical University. All patients were provided written consent before enrollment.

\section{Criteria Of Inclusion And Exclusion}

Patients eligible for enrollment had to meet the following criteria: 1) age $\geq 18$ years, 2) diagnosed with HF according to the guideline3, 3) New York Heart Association (NYHA) functional class II-IV, 4) diagnosis of CHD, and 5) receipt of HF therapy in the past month. Patients were excluded if they experienced acute cardiovascular events in the past 2 months, had a life expectancy of $<1$ year, or refused to participate in this project.

\section{Data Collection}

In-hospital information of patients was collected in accordance with the chronic heart failure case report form (CHF-CRF) that was formulated by our research group according to the contents of case records and the guideline for HF. ${ }^{3}$ CHF-CRF included the patient's age, sex, NYHA class, occupation, health insurance, current smoker, family history, blood pressure, body mass index (BMI), heart rate; presence of diabetes, hypertension, atrial fibrillation, renal insufficiency; laboratory test results. Health insurance was divided into urban and rural health insurance according to the national policies of People's Republic of China based on the patient's occupation and residence. Urban health insurance covers about $80 \%$ of hospitalization expenses, whereas rural health insurance covers only $60 \%$. Indicators for echocardiography and electrocardiography (ECG) performed in hospital were also recorded on the CHF-CRF. Echocardiography was recorded along with standard and tissue Doppler imaging. LVEF was quantified by Simpson's method. Early (E) and late (A) diastolic mitral inflow velocities were measured. E/A values of $<1$ or $>2$ indicated diastolic dysfunction. QRS duration and time of QT were measured manually from the limb leads using standard 12-lead ECG $(25 \mathrm{~mm} / \mathrm{s})$. Information on therapy including drug therapy and percutaneous coronary intervention (PCI) was also recorded on the CHF-CRF.

All patients were followed up routinely at 6-month intervals after discharge by telephone until December 31 , 2018. The telephone follow-up was conducted by trained specialists to record HF re-hospitalization information. When patients were reported to be involved in readmission events, the CHF-CRF form was used to collect corresponding information from the medical records.

\section{Statistical Analysis}

Continuous variables are presented as means \pm standard deviations (SD) and 95\% confidence intervals (CIs) or median \pm interquartile range. Categorical variables are expressed as proportions. Continuous variables were compared using independent $t$-tests for normality distribution and rank-sum test for non-normality distribution. The chisquare test was used to compare the rates.

Cumulative re-hospitalization rates for various time periods were derived from the number of re-hospitalized patients during the period divided by the total number of patients. The probability of hospitalization was plotted using the cumulative incidence function. Time was measured from one hospital discharge to the next admission.

"Listwise deletion" was adopted for missing values. We deleted variables that missing more than $15 \%$. In addition, we added the missing data with missForest.

To evaluate HF re-hospitalization for enrolled patients, we set HF readmission as the dependent variable and indicators of CHF-CRF mentioned above as independent variables to establish Cox regression model. The repeatability and counts of rehospitalization were further analyzed in our study. When the data of repeated hospitalizations were involved, the Cox regression model was no longer suitable, mainly because the data for the repeated hospitalization did not meet the requirement of the Cox model for individual independence. Hence, we introduced the conditional frailty model to further evaluate repeated HF readmissions. ${ }^{9}$ The $k$ times of hospitalization risk of individual $i$ was obtained as follows:

$$
\begin{aligned}
h_{i k}(t) & =h_{0 k}\left(t-t_{k-1}\right) \exp \left(X_{i k} \beta+\varpi_{i}\right) \\
& =u_{i} h_{0 k}\left(t-t_{k-1}\right) \exp \left(X_{i k} \beta\right)
\end{aligned}
$$

$h_{0 k}$ is a baseline hazard function and explains the dependence of events. $u_{i}=\exp \left(\varpi_{i}\right)$ represents the random effect of $i$ and explain the heterogeneity between individuals. 
Epidata 3.1 was applied for double entry of CHF-CRF. Statistical significance was based on two-tailed $P<0.05$. The analyses were undertaken using SPSS version 22.0 and $\mathrm{R}$ software version 3.5.0. The code of $\mathrm{R}$ for conditional frailty model is shown as Supplementary 1 .

\section{Results}

\section{Baseline Characteristics}

Ninety-four patients failed to meet the inclusion criterion in the follow-up study, thus the cohort for this analysis consisted of 1390 patients with HF (93.7\%). Baseline characteristics of the patients are shown in Table 1 . Those who had a re-hospitalization event during the follow-up were older, more likely to be nonmanual workers, and had a higher frequency of hypertension. They had lower levels of diastolic blood pressure, but higher percentage of ejection fraction. The frequency of urban health insurance was higher while the frequencies of rural health insurance and self-paying were lower in the re-hospitalization group. They have lower possibility to take cardiac stimulants or beta-blockers.

\section{Re-Hospitalization Rates}

During the period of the follow-up, 434 (31.2\%) of 1390 HF patients had at least one readmission for HF and 181 $(13 \%)$ had no less than two re-hospitalizations. The cumulative readmission rates at 30 days, 6 months, 1 year, and 2 years were, respectively, $1.88 \%$ (95\% CI $1.18-2.59 \%$ ), 9.91\% (95\% CI $8.36-11.46 \%), 17.38 \%$ (95\% CI $15.41-$ $19.34 \%$ ), and $24.44 \%$ (95\% CI 24.44-29.02\%).

\section{The Incidence Rate Of Re-Hospitalization}

The cumulative incidence function is used to assess the influence of admission number to the next readmission. The result, as shown in Figure 1, indicated that the incidence of hospitalization significantly shortened the interval length between hospital episodes (measured from hospital discharge to the next hospitalization). The median time was decreased from 784 days for the first hospitalization, to 664 days for the second hospitalization, to 515 days for the third hospitalization, and to 388 days for the fourth hospitalization. The cumulative incidence of readmission increased with the number of hospitalization.

\section{Risk Factors For HF Re-Hospitalization}

The results of the Cox regression model are shown in Table 2. Patients engaged in nonmanual work and those with diastolic dysfunction were associated with an increased risk of HF readmission. The treatment of betablockers reduced the risk. Patients with rural health insurance or self-paying had the decreased risk of readmission compared with those with urban health insurance.

To further assess repeated re-hospitalization as timeevent-count data, we utilized the conditional frailty model. Table 2 shows the results of the conditional frailty model. Variables independently increased the risk of HF re-hospitalization were age, female, nonmanual work, longer QRS duration, and treatment with PCI. Rural health insurance and self-paying decreased the risk of readmission compared with urban health insurance. Figure 2 shows the HR and $95 \% \mathrm{CI}$ of each variable.

We analyzed models' behaviour with discrimination ability (C-statistic) and calibration (by a calibration plot). The C-statistic of the Cox regression model and the conditional frailty model were $0.618(P=0.01)$ and 0.892 $(P=0.005)$, respectively as described in Table 2 . The calibration plot is shown in Supplementary 2. Both the models gave good calibration, with points around the first bisector.

The results of proportional hazard $(\mathrm{PH})$ hypothesis tests showed that the data were in coincidence with the conditional risk assumption as shown in Supplementary 3 $(P>0.05)$. We further introduced the Schoenfeld residual diagram to test the $\mathrm{PH}$ hypothesis. The Schoenfeld residuals diagram (Figure 3) showed that the variables and the model met the conditional proportional hazard assumption and were suitable for the conditional frailty model.

\section{Discussion}

In this study, we reported the readmission rate of patients with HF caused by CHD in Shanxi province of People's Republic of China. Compared with previous studies, these parameters allowed us to end up with more accurate, comprehensive results with which to evaluate HF readmission by simultaneously considering the times, events, and numbers of readmissions.

The readmission rate of patients with HF stays at a high level. Previous studies reported that the re-hospitalization rates of patients with $\mathrm{HF}$ at 30 days, 6 months, and 1 year after discharge were, respectively, $24 \%, 50 \%$, and $58.4 \% \cdot{ }^{3,4,10} \mathrm{In}$ this study, we observed lower corresponding readmission rates. The reasons for these lower rates were as follows. First, the endpoints of the studies mentioned above were allcause re-hospitalization, while our study only considered HF readmission. The previous study found that more than half of re-hospitalization occurred due to non-HF causes. ${ }^{11}$ The rate 
Table I Baseline Clinical Characteristics And Clinic Profile Of Patients

\begin{tabular}{|c|c|c|c|}
\hline & Without Rehospitalization $(n=956)$ & Rehospitalization $(n=434)$ & $P$ \\
\hline Age & $69.10 \pm \mid 1.31$ & $70.87 \pm 10.57$ & $<0.001$ \\
\hline Female & $307(32.10 \%)$ & 149 (34.30\%) & 0.414 \\
\hline NYHA & & & 0.682 \\
\hline II & $369(38.6 \%)$ & $173(39.9 \%)$ & \\
\hline III & $358(37.4 \%)$ & $152(35.0 \%)$ & \\
\hline IV & $229(24.0 \%)$ & $109(25.1 \%)$ & \\
\hline Nonmanual workers & $658(68.8 \%)$ & $369(85.0 \%)$ & $<0.001$ \\
\hline Health care & & & $<0.001$ \\
\hline City health insurance & $491(5 I .4 \%)$ & $304(70.0 \%)$ & \\
\hline Rural health insurance & $350(36.6 \%)$ & $83(19.1 \%)$ & \\
\hline Self- paying & 115 (12.00\%) & 47 (10.8\%) & \\
\hline Smoking & $425(44.5 \%)$ & $197(45.4 \%)$ & 0.745 \\
\hline Family history & $178(18.60 \%)$ & 79 (18.2\%) & 0.853 \\
\hline Systolic blood pressure $(\mathrm{mmHg})$ & $|3| \pm 20$ & $|3| \pm 20$ & 0.980 \\
\hline Diastolic blood pressure $(\mathrm{mmHg})$ & $79 \pm 12$ & $78 \pm 12$ & 0.031 \\
\hline BMI $\left(\mathrm{kg} / \mathrm{m}^{2}\right)$ & $24.34 \pm 3.45$ & $24.69 \pm 3.49$ & 0.078 \\
\hline Heart rate & $77 \pm 16$ & $73 \pm 16$ & $<0.001$ \\
\hline \multicolumn{4}{|l|}{ Complications } \\
\hline Hypertension & $586(61.3 \%)$ & $299(68.9 \%)$ & 0.006 \\
\hline Diabetes & $328(34.3 \%)$ & 147 (33.9\%) & 0.510 \\
\hline Atrial fibrillation & $232(24.3 \%)$ & $106(24.4 \%)$ & 0.950 \\
\hline Valvular disease & $378(39.5 \%)$ & $155(35.7 \%)$ & 0.174 \\
\hline Renal insufficiency & $24 I(25.2 \%)$ & $|2|(27.9 \%)$ & 0.293 \\
\hline \multicolumn{4}{|l|}{ Tests } \\
\hline Diastolic dysfunction & $678(70.9 \%)$ & $330(76.0 \%)$ & 0.048 \\
\hline LVDD & $55.90 \pm 7.67$ & $55.85 \pm 7.38$ & 0.887 \\
\hline LVEF\% & $45.13 \pm 12.18$ & $47.06 \pm 12.25$ & 0.006 \\
\hline QRS duration (s) & $106.61 \pm 21.86$ & $108.23 \pm 23.73$ & 0.212 \\
\hline Time of QTc (s) & $444.00 \pm 42.81$ & $442.26 \pm 45.11$ & 0.491 \\
\hline Red blood cells $\left(* 10^{12} / \mathrm{L}\right)$ & $4.35 \pm 0.64$ & $4.29 \pm 0.62$ & 0.085 \\
\hline Hemoglobin (g/L) & $|35.9| \pm 20.28$ & $133.93 \pm 17.77$ & 0.081 \\
\hline Platelet $\left(* 10^{9} / \mathrm{L}\right)$ & $|85.07 \pm 59.5|$ & $184.77 \pm 56.23$ & 0.929 \\
\hline Alanine amino transferase $(\mathrm{U} / \mathrm{L})$ & $28.13 \pm 48.96$ & $29.23 \pm 106.80$ & 0.792 \\
\hline Aspartate amino transferase (U/L) & $32.26 \pm 44.31$ & $37.49 \pm 173.18$ & 0.383 \\
\hline Glucagon (mmol/L) & $5.99 \pm 2.39$ & $5.81 \pm 1.97$ & 0.133 \\
\hline Cholesterol (mmol/L) & $4.08 \pm 1.04$ & $3.91 \pm 1.00$ & 0.013 \\
\hline Triglycerides (mmol/L) & $1.49 \pm 0.73$ & $1.44 \pm 0.75$ & 0.233 \\
\hline High-density lipoprotein $(\mu \mathrm{mol} / \mathrm{L})$ & $1.00 \pm 0.27$ & $1.00 \pm 0.29$ & 0.886 \\
\hline Low-density lipoprotein ( $\mu \mathrm{mol} / \mathrm{L})$ & $2.46 \pm 0.82$ & $2.36 \pm 0.83$ & 0.038 \\
\hline Urea (mmol/L) & $7.33 \pm 5.29$ & $7.11 \pm 5.00$ & 0.469 \\
\hline Serum creatinine $(\mathrm{mmol} / \mathrm{L})$ & $91.85 \pm 45.82$ & $93.48 \pm 40.58$ & 0.524 \\
\hline Uric acid (mmol/L) & $405.56 \pm 137.60$ & $404.49 \pm 135.67$ & 0.712 \\
\hline Estimated GFR $\left(\mathrm{mL} / \mathrm{min} / 1.73 \mathrm{~m}^{2}\right)$ & $66.07 \pm 33.04$ & $64.43 \pm 47.21$ & 0.456 \\
\hline Potassium (mmol/L) & $4.09 \pm 0.47$ & $4.12 \pm 0.4 \mid$ & 0.146 \\
\hline
\end{tabular}

(Continued) 
Table I (Continued).

\begin{tabular}{|c|c|c|c|}
\hline & Without Rehospitalization $(n=956)$ & Rehospitalization $(n=434)$ & $P$ \\
\hline Sodium $(\mathrm{mmol} / \mathrm{L})$ & $|39.11 \pm 4.1|$ & $139.49 \pm 3.14$ & 0.091 \\
\hline Lg of NT-proBNP & $3.28 \pm 0.48$ & $3.2 I \pm 0.5 I$ & 0.033 \\
\hline \multicolumn{4}{|l|}{ Therapy } \\
\hline Antiplatelet therapy & $916(95.8 \%)$ & $422(97.2 \%)$ & 0.196 \\
\hline Statins & $844(88.3 \%)$ & $380(87.6 \%)$ & 0.699 \\
\hline Nitrates & $570(59.6 \%)$ & $254(58.5 \%)$ & 0.699 \\
\hline Beta-blocker & $712(74.50 \%)$ & $297(68.4 \%)$ & 0.019 \\
\hline ACEI or ARB & 339 (35.50\%) & $115(35.7 \%)$ & 0.927 \\
\hline Aldosterone antagonist & $754(78.9 \%)$ & 324 (74.7\%) & 0.081 \\
\hline Diuretic & $640(66.9 \%)$ & $297(68.4 \%)$ & 0.583 \\
\hline Cardiac stimulant & $232(24.3 \%)$ & 81 ( $(8.7 \%)$ & 0.020 \\
\hline ECG monitoring & $762(79.7 \%)$ & $319(73.5 \%)$ & 0.010 \\
\hline $\mathrm{PCl}$ & $283(29.6 \%)$ & 148 (34.1\%) & 0.093 \\
\hline
\end{tabular}

Abbreviations: ACEl, angiotensin-converting enzyme inhibitor; ARB, angiotensin receptor inhibitor; BMI, body mass index; BNP, B-type natriuretic peptide; ECG, electrocardiogram; LVDD, left ventricular diastolic diameter; LVEF, left ventricular ejection fraction; NYHA, New York Heart Association functional class; PCI, percutaneous coronary intervention.

of readmission for HF of previous study was similar to our result. $^{11,12}$ Second, the research objects of our study were patients with HF caused by CHD. Krumholz et al found that the HF readmission rate was lowest in these patients compared with those by other causes, such as non-ischemic cardiomyopathy and atrial fibrillation. ${ }^{13}$ At last, data from People's Republic of China currently exhibit a lower readmission rate of patients with HF than the Western countries. ${ }^{13-16}$

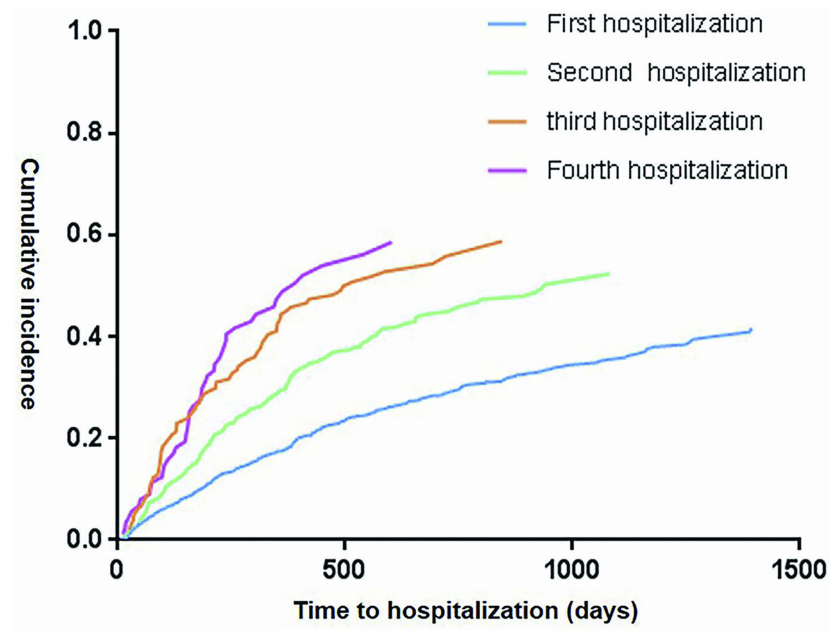

Figure I The cumulative risk of heart failure readmission. Each curve represents the change in the corresponding cumulative incidence of different HF readmission times with the interval between two hospitalizations. The median time decreased from 784 days for the first hospitalization to 664 days for the second hospitalization, 515 days for the third hospitalization, and 388 days for the fourth hospitalization. The cumulative risk of heart failure readmission increased with the number of times of hospitalization.
Repeated occurrences comprised a special feature of rehospitalization. Evaluating the repeatability and number of re-hospitalizations increased the accuracy and rationality of the re-hospitalization assessment. However, as noted, when repeated re-hospitalizations were considered, the Cox regression was no longer suitable. Researchers have been concerned about this point and developed various statistical methods with which to solve this problem. Braga et al used the Prentice-Williams-Peterson (PWP) model with a total all time scale (TT) to identify independent predictors of repeated hospital admissions and revealed that the PWP-TT generated more conservative estimates than the Cox regression model. ${ }^{17}$ Besides the PWP model, naive techniques, variance-corrected models were also used. However, these statistical models mentioned above ignored the heterogeneity or nonindependence of readmission data. To better tackle recurrent data, Oakes et al added the frailty factor to the Cox regression model to form the frailty model. ${ }^{18}$ Ullah et al demonstrated that the frailty model was more suitable for recurrent data compared with the Wei, Lin, and Weissfeld model and the PWP model. ${ }^{19}$ To further improve this model, Box-Steffensmeier et al applied a random effect that could better describe the nonindependence of recurrence data to establish the conditional frailty model. ${ }^{9}$ Therefore, in the present study, we introduced the conditional frailty model to evaluate the $\mathrm{HF}$ readmission as event-time-count data in addition to using the Cox regression model.

According to the results of the Cox regression model, we found that nonmanual workers had a high risk of $\mathrm{HF}$ 
Table 2 Cox Regression Model And Conditional Frailty Model Of Risk Factors For HF Readmission

\begin{tabular}{|l|l|l|l|l|l|l|l|l|l|l|}
\hline \multirow{2}{*}{ Variable } & \multicolumn{4}{l}{ Cox Regression Model } & \multicolumn{3}{l|}{ Conditional Frailty Model } \\
\cline { 2 - 10 } & B & SE & P & HR & HR 95\% CI & B & SE & P & HR & HR 95\% CI \\
\hline Age & - & - & - & - & - & 0.012 & 0.005 & 0.008 & 1.013 & $(1.003,1.022)$ \\
Female & - & - & - & - & - & 0.252 & 0.106 & 0.018 & 1.286 & $(1.045,1.583)$ \\
Non-manual worker & 0.450 & 0.195 & 0.021 & 1.568 & $(1.071,2.295)$ & 0.394 & 0.177 & 0.026 & 1.483 & $(1.049,2.096)$ \\
Rural health insurance & -0480 & 0.180 & 0.007 & 0.619 & $(0.435,0.879)$ & -0.510 & 0.161 & 0.008 & 0.601 & $(0.438,0.824)$ \\
Self-paying & -0.346 & 0.158 & 0.029 & 0.708 & $(0.519,0.965)$ & -0.420 & 0.159 & 0.008 & 0.657 & $(0.481,0.898)$ \\
QRS duration & - & - & - & - & - & 0.004 & 0.002 & 0.022 & 1.004 & $(1.001,1.008)$ \\
Diastolic dysfunction & 0.320 & 0.113 & 0.005 & 1.377 & $(1.104,1.718)$ & - & - & - & - & - \\
Beta-blocker & -0.239 & 0.103 & 0.021 & 0.787 & $(0.643,0.964)$ & - & - & - & - & - \\
PCl & - & - & - & - & - & 0.209 & 0.099 & 0.035 & 1.232 & $(1.015,1.497)$ \\
Frailty gamma (ID) & - & - & - & - & - & 847.19 & 500.4 & $<0.001$ & & \\
C-statistic & & & & & $0.595(0.01)$ & & & & & $0.896(0.005)$ \\
\hline
\end{tabular}

Abbreviations: $\mathrm{HR}$, hazard ration; $\mathrm{Cl}$, confidence interval; $\mathrm{PCl}$, percutaneous coronary intervention.

readmission. The same result was reported in a small prospective cohort study. ${ }^{20}$ This observation is caused possibly by that nonmanual workers could pay more time and energy to their physical condition. Health insurance was an important influencing factor for HF readmission as reported previously. ${ }^{21,22}$ In People's Republic of China, the situation of health insurance is unique. Compared with rural health insurance, patients with urban health insurance are able to receive better medical compensation from medical insurance agencies. Our study showed that patients with urban health insurance (with its better medical compensation) were at a higher risk of readmission than those with rural health insurance or who were self-paying. This result suggested that some patients may be hospitalized for economic reasons rather than their disease. This possibility calls for further

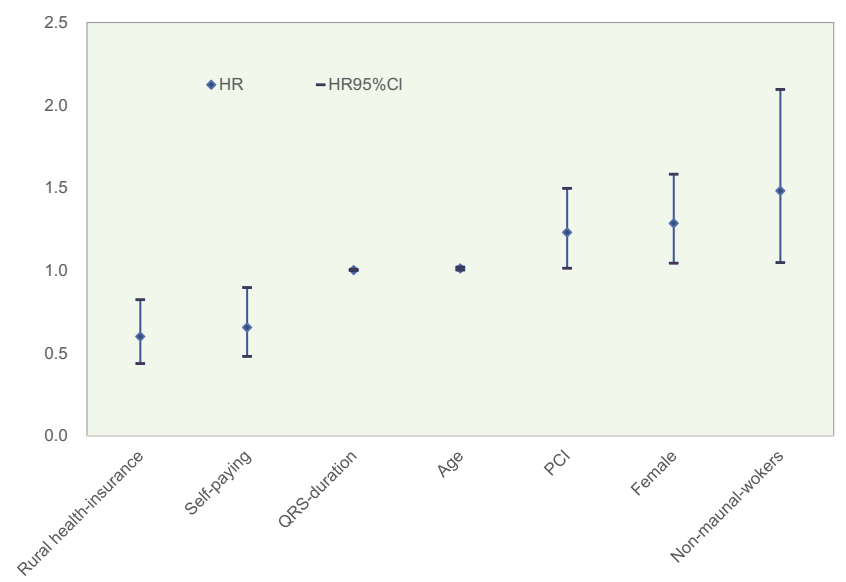

Figure 2 HRs of risk factors for heart failure readmission analyzed by conditional frailty model. Plots represent $\mathrm{HRs}$ of the risk factors and bars indicate $95 \% \mathrm{Cl}$. Abbreviations: $\mathrm{HR}$, hazard ratio; $\mathrm{PCl}$, percutaneous coronary intervention. research to confirm this result and to identify the most suitable health insurance for patients and the national economy. Our study also confirmed that the use of beta-blockers decreased the risk of HF re-hospitalization, which is consistent with previous studies. ${ }^{23,24}$ Furthermore, our study showed that diastolic dysfunction increased the risk of $\mathrm{HF}$ readmission. Diastolic dysfunction is the main cause of $\mathrm{HF}$ with preserved ejection fraction (HFpEF). Studies have proved that patients with $\mathrm{HFpEF}$ had a similar prognosis to those with reduced ejection fraction. ${ }^{25,26}$ The increased influence of diastolic dysfunction in our study may be attributed to $\mathrm{CHD}$ as an independent risk factor for $\mathrm{HFpEF}$, as presented in previous research. ${ }^{27}$ We should pay more attention to improve diastolic dysfunction of patients with CHD.

The conditional frailty model was further used to analyze HF re-hospitalizations as time-event-count data, with the goal of obtaining more accurate and reasonable results. ${ }^{9}$ Nonmanual workers and those with better medical compensation were still at an increased risk of HF readmission when recurrent readmissions were considered. Moreover, we found that aging increased the risk of readmission. Elderly patients had poor outcomes, as indicated by previous researches. ${ }^{28-30}$ Elderly patients with HF have poorer physiological function, more complications, and lower compliance with therapy, and all of them contribute to the increase in HF re-hospitalization. With regard to gender, our study showed that women were more likely to be readmitted for HF compared with men. A large-scale research consisting of 1,392,289 patients with HF reported an adjusted HR of 1.041 for HF readmission in female patients. ${ }^{31}$ However, Rørth et al reported the opposite conclusion in the population of patients aged $18-60$ years. ${ }^{32}$ 
Schoenfeld Individual Test p: 0.5094
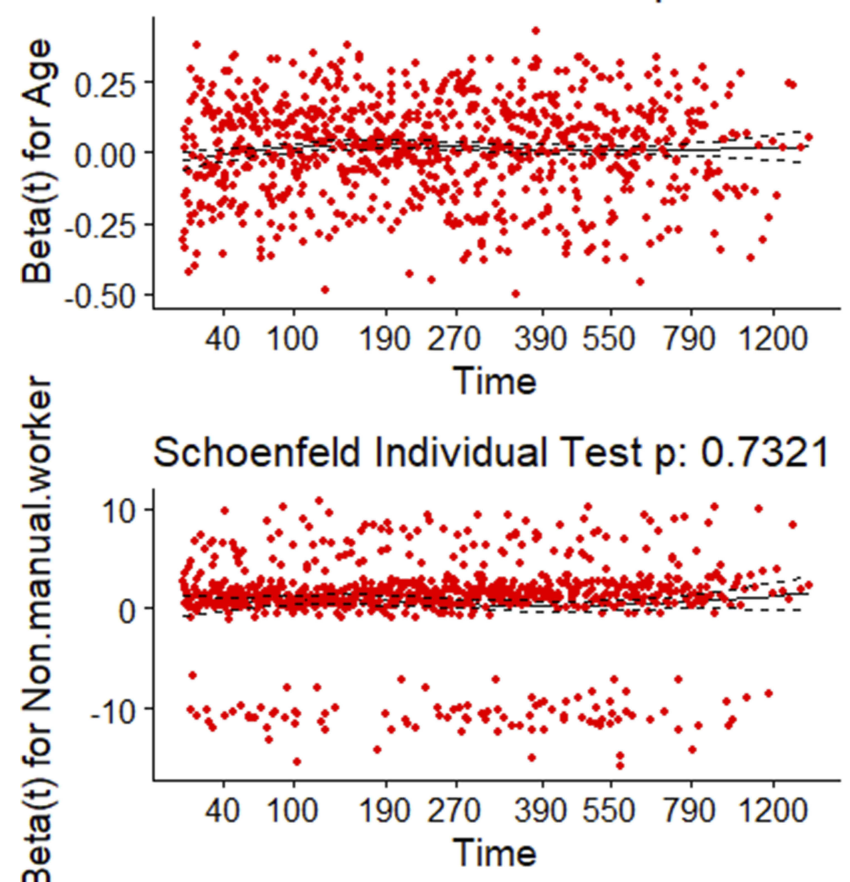

Schoenfeld Individual Test p: 0.3033
告

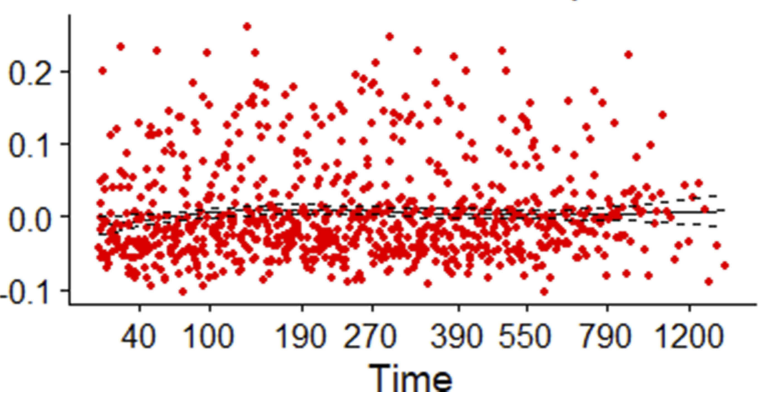

Schoenfeld Individual Test p: 0.904
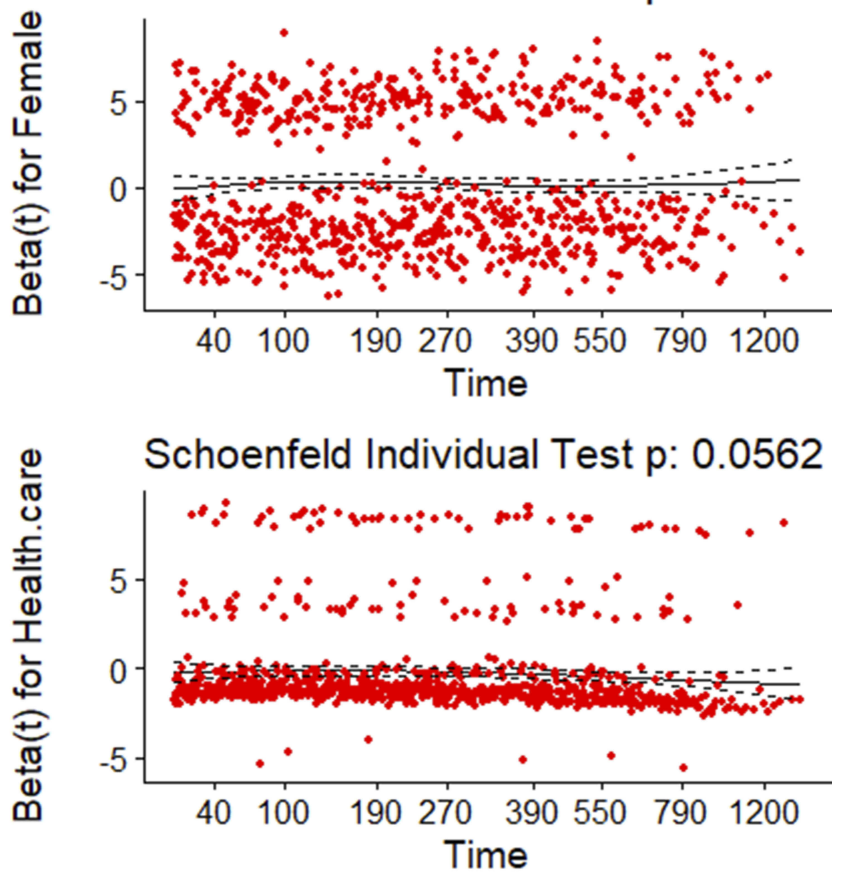

Schoenfeld Individual Test p: 0.0746

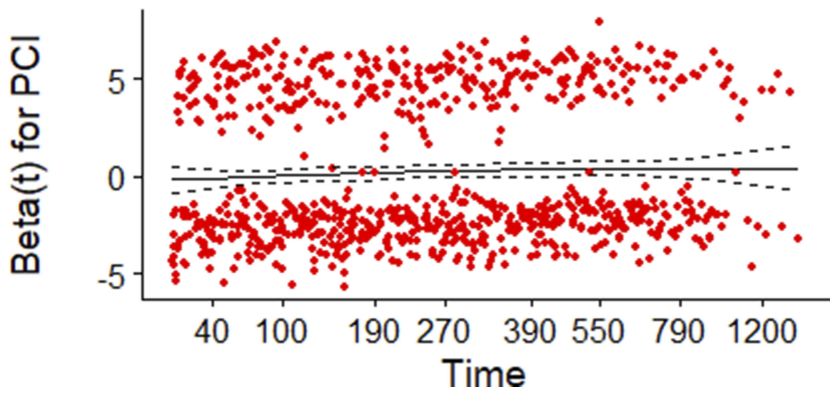

Figure 3 Schoenfeld residual diagram. The curve of each diagram represents the trend of risk factor change as time goes by. The $P$-values of the variables and the model as a whole were shown in the diagram. Survminer, Survival, ggplot2, and other packages of R3.5.0 and related programs were used to create the Schoenfeld residuals graph. $P>0.05$ indicates that the variable meets the conditional proportional hazard assumption.

Abbreviation: $\mathrm{PCl}$, percutaneous coronary intervention.

The main cause of the difference might be the age of the patients included in the studies. The next step might be to consider the influence of the patient's sex on readmissions as the body changes with age. The QRS duration could be prolonged by myocardial remodeling which is the performance of HF. Studies have shown that prolonged QRS duration was associated with an increased incidence of cardiac events, including readmission, ${ }^{33-36}$ particularly in patients with ischemic heart disease. ${ }^{34}$ Our study also found the same conclusion. We believe that PCI could increase the coronary blood supply and improve the prognosis of HF. Our study showed that PCI increased the risk of HF readmission, which was consistent with a previous finding. ${ }^{37}$ This phenomenon may be related to incomplete revascularization, more follow-up visits, and the side effects of drugs. Clinical research on the relation between PCI and $\mathrm{HF}$ is still a rarity, and further study is required, as recommended in the European Society of Cardiology guidelines. ${ }^{38}$ The reason for this finding is not clear, and it deserves clinical consideration and further research.

There are several important limitations to this analysis. First, the patients of this study were all from Shanxi province in People's Republic of China. Hence, they did not represent all Chinese people (or other nationalities). 
Future prospective studies with a larger patient population are required. Second, some important patient information was not collected because of incomplete medical records. As a result, many important characteristics were not included in the models. Last, this study failed to cover all of the statistical methods for recurrent data. We will further study the methods and introduce them in the next research.

\section{Conclusions}

In conclusion, our study analyzed the time-event data and the time-event-count data of re-hospitalization to evaluate the HF re-hospitalization more comprehensively, accurately and reasonably for patients with $\mathrm{HF}$ caused by CHD. Both models showed good calibration and the conditional frailty model performance higher discrimination ability compared with the Cox model, so this finding provides more clinical epidemiological evidence which allow us to distinguish patients at a high risk of re-hospitalization more accurately, so as to guide precise and personalized management of heart failure.

\section{Acknowledgments}

We are grateful for the cooperation of Shanxi Cardiovascular Hospital. Thanks for the funding of the National Natural Science Foundation of China (grant no. 81872714), the General Program for Young Scholar of Shanxi Province (grant no. 201801D221423), and Scientific Research Project of Shanxi Provincial Health Committee (grant nos. 2017038 and 2018039).

\section{Author Contributions}

All authors contributed to data analysis, drafting or revising the article, gave final approval of the version to be published, and agree to be accountable for all aspects of the work.

\section{Disclosure}

The authors report no conflicts of interest in this work.

\section{References}

1. Arnett DK, Blumenthal RS, Albert MA, et al. ACC/AHA guideline on the primary prevention of cardiovascular disease: executive summary. Circulation. 2019;2019:e1-171.

2. Yaku H, Ozasa N, Morimoto T, et al. Demographics, management, and in-hospital outcome of hospitalized acute heart failure syndrome patients in contemporary real clinical practice in japan-observations from the prospective, multicenter Kyoto Congestive Heart Failure (KCHF) registry. Circ J. 2018;82(11):2811-2819. doi:10.1253/circj. CJ-17-1386
3. Yancy CW, Jessup M, Bozkurt B, et al. 2017 ACC/AHA/HFSA focused update of the $2013 \mathrm{ACCF} / \mathrm{AHA}$ guideline for the management of heart failure: a report of the American College of Cardiology/American Heart Association Task Force on Clinical Practice Guidelines and the Heart Failure Society of America. Circulation. 2017;136(6):e137-e161. doi:10.1161/CIR.0000000000000509

4. Gheorghiade M, Vaduganathan M, Fonarow GC, Bonow RO. Rehospitalization for heart failure: problems and perspectives. $J \mathrm{Am}$ Coll Cardiol. 2013;61(4):391-403. doi:10.1016/j.jacc.2012.09.038

5. Fonarow GC. Clinical risk prediction tools in patients hospitalized with heart failure. Rev Cardiovasc Med. 2012;13(1):e14-e23.

6. Giamouzis G, Kalogeropoulos A, Georgiopoulou V, et al. Hospitalization epidemic in patients with heart failure: risk factors, risk prediction, knowledge gaps, and future directions. J Card Fail. 2011;17(1):54-75. doi:10.1016/j.cardfail.2010.08.010

7. Therneau T, Grambsch P. Modeling survival data: extending the cox model. Technometrics. 2000;44(1):85-86.

8. Therneau T, Pankratz P. Penalized survival models and frailty. J Comput Graphical Stat. 2003;12(1):156-175. doi:10.1198/1061860031365

9. Box-Steffensmeier JM, De Boef S. Repeated events survival models: the conditional frailty model. Stat Med. 2006;25(20):e1-e12. doi:10.1002/(ISSN)1097-0258

10. Dunlay SM, Redfield MM, Weston SA, et al. Hospitalizations after heart failure diagnosis: a community perspective. $\mathrm{J} \mathrm{Am} \mathrm{Coll} \mathrm{Cardiol.}$ 2009;54(18):1695-1702. doi:10.1016/j.jacc.2009.08.019

11. Fudim M, O'Connor CM, Dunning A, et al. Aetiology, timing and clinical predictors of early vs. late readmission following index hospitalization for acute heart failure: insights from ASCEND-HF. Eur J Heart Fail. 2018;20(2):304-314. doi:10.1002/ejhf.2018.20. issue-2

12. Bottle A, Aylin P, Bell D. Effect of the readmission primary diagnosis and time interval in heart failure patients: analysis of English administrative data. Eur J Heart Fail. 2014;16(8):846-853. doi:10.1002/ ejhf.2014.16.issue-8

13. Krumholz HM, Dhruva S. Real-world data on heart failure readmission reduction: real or real uncertain? J Am Coll Cardiol. 2017;69 (19):2366-2368. doi:10.1016/j.jacc.2017.03.019

14. Minghua C, Boqing O, Sidan D, Yuanyuan Y. Influence factors of rehospitalization in 797 patients with chronic heart failure. Med Clin Res. 2013;30(7):436-438. China.

15. Can Z, Wei G, Xiang T, et al. Risk factors of re-hospitalization in patients with heart failure within 6 months. Medical Rev. 2018;24 (6):2275-2280. China.

16. Min X, Jincheng G, Lixin Z, Ming G, Haibin Z. Analysis of CHF treatment status and influence factors of re-hospitalization in Tongzhou, Beijing. Chin J Evidence-Based Cardiovasc Med. 2017;9 (11):569-574. China.

17. Braga JR, Tu JV, Austin PC, Sutradhar R, Ross HJ, Lee DS. Recurrent events analysis for examination of hospitalizations in heart failure: insights from the Enhanced Feedback for Effective Cardiac Treatment (EFFECT) trial. Eur Heart J Qual Care Clin Outcomes. 2018;4(1):18-26. doi:10.1093/ehjqcco/qcx015

18. Oakes D. Frailty models for multiple event times. Survival Anal. 1992;371-379.

19. Ullah S, Gabbett TJ, Finch CF. Statistical modelling for recurrent events: an application to sports injuries. Br J Sports Med. 2014;48 (17):1287-1293. doi:10.1136/bjsports-2011-090803

20. Noori A, Shokoohi M, Baneshi MR, Naderi N, Bakhshandeh H, Haghdoost AA. Impact of socio-economic status on the hospital readmission of congestive heart failure patients: a prospective cohort study. Int J Health Policy Manag. 2014;3(5):251-257. doi:10.15171/ ijhpm.2014.94

21. Philbin EF, DiSalvo TG. Prediction of hospital readmission for heart failure: development of a simple risk score based on administrative data. J Am Coll Cardiol. 1999;33(6):1560-1566. doi:10.1016/S07351097(99)00059-5 
22. Allen LA, Smoyer Tomic KE, Smith DM, Wilson KL, Agodoa I. Rates and predictors of 30-day readmission among commercially insured and Medicaid-enrolled patients hospitalized with systolic heart failure. Circ Heart Fail. 2012;5(6):672-679. doi:10.1161/ CIRCHEARTFAILURE.112.967356

23. Yamaguchi T, Kitai T, Miyamoto T, et al. Effect of optimizing guideline-directed medical therapy before discharge on mortality and heart failure readmission in patients hospitalized with heart failure with reduced ejection fraction. Am J Cardiol. 2018;121(8):969-974. doi:10.1016/j.amjcard.2018.01.006

24. Nakatani D, Sakata Y, Mizuno H, et al. Impact of diabetes mellitus on re-hospitalization for heart failure among survivors of acute myocardial infarction in the percutaneous coronary intervention era. Circ J. 2009;73(4):662-666. doi:10.1253/circj.CJ-08-0579

25. Badano LP, Albanese MC, De Biaggio P, et al. Prevalence, clinical characteristics, quality of life, and prognosis of patients with congestive heart failure and isolated left ventricular diastolic dysfunction. J Am Soc Echocardiogr. 2004;17(3):253-261.

26. Miyagishima K, Hiramitsu S, Kimura H, et al. Long-term prognosis of chronic heart failure: reduced vs preserved left ventricular ejection fraction. Circ J. 2009;73(1):92-99. doi:10.1253/circj.CJ-07-1016

27. McMurray JJ, Adamopoulos S, Anker SD, et al. ESC guidelines for the diagnosis and treatment of acute and chronic heart failure 2012 the task force for the diagnosis and treatment of acute and chronic heart failure 2012 of the European Society of Cardiology. Developed in collaboration with the Heart Failure Association (HFA) of the ESC. Eur J Heart Fail. 2012;14(8):803-869. doi:10.1093/eurjhf/ hfs 105

28. Pacho C, Domingo M, Núñez R, et al. Predictive biomarkers for death and re-hospitalization in comorbid frail elderly heart failure patients. BMC Geriatr. 2018;18(1):e1-e10. doi:10.1186/s12877-018-0807-2

29. Ruigómez A, Michel A, Martín-Pérez M, García Rodríguez LA. Heart failure hospitalization: an important prognostic factor for heart failure re-admission and mortality. Int $J$ Cardiol. 2016;220:855-861. doi:10.1016/j.ijcard.2016.06.080
30. Kaneko H, Suzuki S, Goto M, et al. Incidence and predictors of rehospitalization of acute heart failure patients. Int Heart J. 2015;56:219-225. doi:10.1536/ihj.14-290

31. Dreyer RP, Dharmarajan K, Hsieh AF, Welsh J, Qin L, Krumholz HM. Sex differences in trajectories of risk after re-hospitalization for heart failure, acute myocardial infarction, or pneumonia. Circ Cardiovasc Qual Outcomes. 2017;10(5):e1-e10. doi:10.1161/ CIRCOUTCOMES.116.003271

32. Rørth R, Fosbøl EL, Mogensen UM, et al. Employment status at time of first hospitalization for heart failure is associated with a higher risk of death and re-hospitalization for heart failure. Eur J Heart Fail. 2018;20(2):240-247. doi:10.1002/ejhf.2018.20.issue-2

33. Peterson PN, Greiner MA, Qualls LG, et al. QRS duration, bundlebranch block morphology, and outcomes among older patients with heart failure receiving cardiac resynchronization therapy. JAMA. 2013;310(6):617-626. doi:10.1001/jama.2013.8641

34. Park HS, Kim H, Park JH, et al. QRS prolongation in the prediction of clinical cardiac events in patients with acute heart failure: analysis of data from the Korean Acute Heart Failure Registry. Cardiology. 2013;125(2):96-103. doi:10.1159/000348334

35. Gouda P, Brown P, Rowe BH, McAlister FA, Ezekowitz JA. Insights into the importance of the electrocardiogram in patients with acute heart failure. Eur J Heart Fail. 2016;18(8):1032-1040. doi:10.1002/ ejhf.561

36. Danciu SC, Gonzalez J, Gandhi N, Sadhu S, Herrera CJ, Kehoe R. Comparison of six-month outcomes and hospitalization rates in heart failure patients with and without preserved left ventricular ejection fraction and with and without intraventricular conduction defect. $\mathrm{Am}$ J Cardiol. 2006;97(2):256-259. doi:10.1016/j.amjcard.2005.08.031

37. Carey SA, Bass K, Saracino G, et al. Probability of accurate heart failure diagnosis and the implications for hospital readmissions. Am J Cardiol. 2017;119(7):1041-1046. doi:10.1016/j.amjcard.2016.12.010

38. Ponikowski P, Voors AA, Anker SD, et al. 2016 ESC guidelines for the diagnosis and treatment of acute and chronic heart failure. Rev Esp Cardiol (Engl Ed). 2016;69(12):1037-1147.
Therapeutics and Clinical Risk Management

\section{Publish your work in this journal}

Therapeutics and Clinical Risk Management is an international, peerreviewed journal of clinical therapeutics and risk management, focusing on concise rapid reporting of clinical studies in all therapeutic areas, outcomes, safety, and programs for the effective, safe, and sustained use of medicines. This journal is indexed on PubMed Central, CAS,
EMBase, Scopus and the Elsevier Bibliographic databases. The manuscript management system is completely online and includes a very quick and fair peer-review system, which is all easy to use. Visit http://www.dovepress.com/testimonials.php to read real quotes from published authors. 